\title{
An Asymptotic Expansion for the Number of Permutations with a Certain Number of Inversions
}

\author{
Lane Clark \\ Department of Mathematics \\ Southern Illinois University Carbondale \\ Carbondale, IL 62901-4408 USA \\ lclark@math.siu.edu
}

Submitted: December 17, 1998; Accepted: August 8, 2000

\begin{abstract}
Let $b(n, k)$ denote the number of permutations of $\{1, \ldots, n\}$ with precisely $k$ inversions. We represent $b(n, k)$ as a real trigonometric integral and then use the method of Laplace to give a complete asymptotic expansion of the integral. Among the consequences, we have a complete asymptotic expansion for $b(n, k) / n$ ! for a range of $k$ including the maximum of the $b(n, k) / n$ !.
\end{abstract}

AMS Subject Classification: 05A16, 05A15, 05A10

A permutation $\sigma=(\sigma(1), \ldots, \sigma(n))$ of $[n]=\{1, \ldots, n\}$ has an inversion at $(i, j)$, where $1 \leq i<j \leq n$, if and only if $\sigma(i)>\sigma(j)$. Let $b(n, k)$ denote the number of permutations of $[n]$ with precisely $k$ inversions. Then $b(n, k)=b\left(n,\left(\begin{array}{l}n \\ 2\end{array}\right)-k\right)$ for all integers $k$, while, $b(n, k) \neq 0$ if and only if $0 \leq k \leq\left(\begin{array}{l}n \\ 2\end{array}\right)$. Bender [2; p. 110] showed that the $b(n, k)$ are log concave in $k$. Hence, the maximum $B(n)$ of the $b(n, k)$ occurs at $k=\left\lfloor\left(\begin{array}{l}n \\ 2\end{array}\right) / 2\right\rfloor$, as well as $\left\lceil\left(\begin{array}{l}n \\ 2\end{array}\right) / 2\right\rceil$ for odd $\left(\begin{array}{l}n \\ 2\end{array}\right)$. See [3; pps. 236-240] for further results.

Random permutations show (see [3; pps. 282-283], for example) that the $b(n, k)$ satisfy a central limit theorem with $\mu_{n}=\left(\begin{array}{c}n \\ 2\end{array}\right) / 2$ and $\sigma_{n}^{2}=n(n-1)(2 n+5) / 72$ (see [2; Theorem 1]). Bender [2; p. 109] remarks that "the theorems of Section 4 do not apply" to the $b(n, k)$. He then shows $[2 ;$ p. 110] that the $b(n, k)$ are log concave in $k$ "so that Lemma 2 applies." This will give a (first term) asymptotic formula for $b(n, k) / n$ ! when $k=\left\lfloor\mu_{n}+x \sigma_{n}\right\rfloor$ where $x$ is a fixed real number.

In this paper, we represent $b(n, k)$ as a real trigonometric integral. We then use the method of Laplace to give a complete asymptotic expansion of this integral in terms of the Bernoulli numbers and Hermite polynomials. Hence, we have the complete asymptotic 
expansion

$$
\begin{aligned}
\frac{b(n, k)}{n !}=6(2 \pi)^{-1 / 2} n^{-3 / 2} \mathrm{e}^{-x^{2} / 2}\left\{1+\sum_{q=1}^{2 m-2}(-2)^{-q} S_{2 q}(n) H_{2 q}\left(2^{-1 / 2} x\right)\right\} \\
+O\left(\frac{\ln ^{2 m^{2}+1} n}{n^{m+3 / 2}}\right) \text { as } n \rightarrow \infty,
\end{aligned}
$$

when $2 k=\left(\begin{array}{l}n \\ 2\end{array}\right) \pm x n^{3 / 2} / 3$ where $x^{2}=x^{2}(n) \leq \ln n$ and $m$ is a fixed integer at least 2 . Here, $H_{2 q}$ are the Hermite polynomials defined before Theorem 1 and the $S_{2 q}$ are defined in Theorem 3. In particular, we have a complete asymptotic expansion for $B(n) / n$ ! when $\left(\begin{array}{l}n \\ 2\end{array}\right)$ is even. See Corollaries 2, 4 for other asymptotic expansions.

In what follows, $k, \ell$ and $n$ are integers with $0 \leq k \leq\left(\begin{array}{l}n \\ 2\end{array}\right)$ and $2 \leq \ell \leq n$. We denote the nonnegative integers by $\mathbb{N}$. All asymptotic formulas are for $n \rightarrow \infty$.

Muir [5] (see also [3; p. 239]) showed that $b(n, k)$ is the coefficient of $z^{k}$ in $\prod_{\ell=2}^{n}(1+$ $\left.z+\cdots+z^{\ell-1}\right)$. Then,

$$
\begin{aligned}
b(n, k) & =\frac{1}{2 \pi i} \oint_{C} \frac{\prod_{\ell=2}^{n}\left(1+z+\cdots+z^{\ell-1}\right)}{z^{k+1}} d z \\
& =\frac{1}{2 \pi i} \oint_{C} z^{-k-1} \prod_{\ell=2}^{n}\left(\frac{z^{\ell}-1}{z-1}\right) d z
\end{aligned}
$$

where $C$ is the unit circle. Hence,

$$
b(n, k)=\frac{2 n !}{\pi} \int_{0}^{\pi / 2} \prod_{\ell=2}^{n}\left(\frac{\sin \ell t}{\ell \sin t}\right) \cos \left(\left(\left(\begin{array}{c}
n \\
2
\end{array}\right)-2 k\right) t\right) d t
$$

upon parameterizing $C\left(z=\mathrm{e}^{i t} ; t \in[0,2 \pi]\right)$ and using the symmetry of the integrand.

For an integer $n \geq 2$ and real numbers $a, b$ and $x$, let

$$
I(n, x, a, b):=\int_{a}^{b} \prod_{\ell=2}^{n}\left(\frac{\sin \ell t}{\ell \sin t}\right) \cos \left(\frac{x t n^{3 / 2}}{3}\right) d t
$$

and

$$
I(n, x):=I\left(n, x, 0, \frac{\pi}{2}\right)
$$

(where all discontinuities of the integrand have been removed). Then (2) gives

$$
\frac{b(n, k)}{n !}=\frac{2}{\pi} I(n, x)
$$

for all integers $k, n$ where $0 \leq k \leq\left(\begin{array}{l}n \\ 2\end{array}\right), n \geq 2$ and $2 k=\left(\begin{array}{l}n \\ 2\end{array}\right) \pm x n^{3 / 2} / 3$. 
For a nonnegative integer $q$ and real number $x$, let

$$
F_{q}(x):=\int_{0}^{\infty} \exp \left(-u^{2} / 2\right) u^{q} \cos (u x) d u
$$

denote the Fourier cosine transform of $\exp \left(-u^{2} / 2\right) u^{q}$. Then $F_{2 q}(x)=$ $(-1)^{q} \pi^{1 / 2} 2^{-q-1 / 2} \mathrm{e}^{-x^{2} / 2} H_{2 q}\left(2^{-1 / 2} x\right)$. Here $H_{n}(x)$ are the Hermite polynomials given by $H_{n}(x)=\sum_{k=0}^{\lfloor n / 2\rfloor}(-1)^{k} n !(2 x)^{n-2 k} / k !(n-2 k) ! \quad$ (see [4; pps. 60-64]).

We use the following Taylor series approximations which are valid for all real numbers $t$.

$$
\begin{gathered}
\sin t=t-\frac{t^{3}}{6}+a(t) ; \quad|a(t)| \leq \frac{t^{4}}{24} \text { for all real } t \text { and } a(t) \geq 0 \text { for } t \in[0, \pi] ; \\
\cos t=1-\frac{t^{2}}{2}+b(t) ; \quad 0 \leq b(t) \leq t^{3} \text { for } t \in[0, \pi] ;
\end{gathered}
$$

and for an integer $m \geq 1$,

$$
\mathrm{e}^{t}=1+t+\cdots+\frac{t^{m-1}}{(m-1) !}+c_{m}(t) ; \quad\left|c_{m}(t)\right| \leq \mathrm{e}^{|t|}|t|^{m} .
$$

Of course, our error terms $a, b$ and $c_{m}$ are all infinitely-differentiable functions over the reals. We also require the following inequality (integration by parts). For a real number $x>0$,

$$
\int_{x}^{\infty} \mathrm{e}^{-t^{2} / 2} d t \leq \frac{1}{x} \mathrm{e}^{-x^{2} / 2}
$$

We now give our first result.

Theorem 1. For $x^{2}=x^{2}(n) \leq \ln n$, we have the asymptotic expansion

$$
\begin{aligned}
I(n, x)=3 & \left(\frac{\pi}{2}\right)^{1 / 2} n^{-3 / 2} e^{-x^{2} / 2}\left\{1-\frac{1}{100 n}\left(9 x^{4}-129 x^{2}+102\right)\right. \\
& +\frac{1}{980000 n^{2}}\left(3969 x^{8}-141282 x^{6}+1340865 x^{4}\right. \\
& \left.\left.-4579480 x^{2}+2259370\right)\right\}+O\left(\frac{\ln ^{19} n}{n^{9 / 2}}\right) \text { as } n \rightarrow \infty .
\end{aligned}
$$

Proof. We use the method of Laplace. For $0<a \leq 1$ and an integer $\ell \geq 2$, let $M_{\ell}(a):=\max \{|\sin \ell t / \sin t|: t \in[a, \pi / 2]\}$ and $b:=\cos a \in(0,1)$. For all integers $\ell \geq 2$, $M_{\ell}(a) \leq b^{\ell-1}+b^{\ell-2}+\cdots+b+1 \leq \min \left\{\ell,(1-b)^{-1}\right\}$ by induction on $\ell$, while $a^{2} / 3 \leq 1-b$. Here,

$$
\prod_{\ell=2}^{n}\left|\frac{\sin \ell t}{\ell \sin t}\right| \leq \frac{(1-b)^{-n}}{n !} \leq\left(\frac{3 \mathrm{e}}{a^{2} n}\right)^{n}
$$


and, hence, for all $n \geq 9$ and all real numbers $x$,

$$
\left|I\left(n, x, 3 n^{-0.5}, \pi / 2\right)\right| \leq 2\left(\frac{\mathrm{e}}{3}\right)^{n} .
$$

For all integers $\ell$ and all real numbers $t$ with $\sin t \neq 0$, (4) gives $\sin \ell t / \ell \sin t=$ $1-\left(\ell^{2}-1\right) t^{2} / 6+d(\ell, t)$ where $|d(\ell, t)| \leq \ell^{3} t^{3} / 12$ for $t \in(0,1]$ and $\ell \geq 2$. Hence,

$$
0<\frac{\sin \ell t}{\ell \sin t} \leq 1-\frac{\ell^{2} t^{2}}{24} \leq \exp \left(-\frac{\ell^{2} t^{2}}{24}\right) \text { for } \ell t \in[0,1] \text { and } \ell \geq 2 .
$$

(Naturally, we define $\sin \ell t / \ell \sin t=1$ when $t=0$ to remove that discontinuity.) For all $n \geq 144$ and all real numbers $x,(9)$ gives

$$
\begin{gathered}
\left|I\left(n, x, n^{-0.7}, 3 n^{-0.5}\right)\right| \leq \int_{n^{-0.7}}^{3 n^{-0.5}} \prod_{\ell=2}^{\left\lfloor n^{0.5} / 3\right\rfloor} \frac{\sin \ell t}{\ell \sin t} d t \leq \exp \left(\frac{-n^{0.1}}{4608}\right), \\
\left|I\left(n, x, n^{-1}, n^{-0.7}\right)\right| \leq \int_{n^{-1}}^{n^{-0.7}} \prod_{\ell=2}^{\left\lfloor n^{0.7}\right\rfloor} \frac{\sin \ell t}{\ell \sin t} d t \leq \exp \left(\frac{-n^{0.1}}{576}\right),
\end{gathered}
$$

and

$$
\left|I\left(n, x, n^{-3 / 2} \ln n, n^{-1}\right)\right| \leq \int_{n^{-3 / 2} \ln n}^{n^{-1}} \prod_{\ell=2}^{n} \frac{\sin \ell t}{\ell \sin t} d t \leq \exp \left(-\frac{\ln ^{2} n}{72}\right)
$$

Recall that $\cot t=t^{-1}+\sum_{k=1}^{\infty}(-4)^{k} B_{2 k} t^{2 k-1} /(2 k)$ !, for real $t$ with $0<|t|<\pi$. Here $B_{n}$ are the Bernoulli numbers defined by $z /\left(\mathrm{e}^{z}-1\right)=\sum_{n=0}^{\infty} B_{n} z^{n} / n$ ! for complex $z$ with $|z|<2 \pi$ (see [3; pps. 48, 88]). Then, $\frac{d}{d t}\{\ln (\sin \ell t / \ell \sin t)\}=\ell \cot \ell t-\cot t=$ $\sum_{k=1}^{\infty}(-4)^{k} B_{2 k}\left(\ell^{2 k}-1\right) t^{2 k-1} /(2 k)$ ! for $0<|\ell t|<\pi$, hence,

$$
\ln \left(\frac{\sin \ell t}{\ell \sin t}\right)=\sum_{k=1}^{\infty}(-4)^{k} B_{2 k}\left(\ell^{2 k}-1\right) \frac{t^{2 k}}{(2 k)(2 k) !} \text { for }|\ell t|<\pi .
$$

For a nonnegative integer $m,|\ell t| \leq 1$ and $\ell \geq 1$ (see [1; p. 805]),

$$
\left|\sum_{k=m+1}^{\infty}(-4)^{k} B_{2 k}\left(\ell^{2 k}-1\right) \frac{t^{2 k}}{(2 k)(2 k) !}\right| \leq \ell^{2 m+2} t^{2 m+2} \text {. }
$$

For $n \geq 2$ and $\theta_{k}(n):=\sum_{\ell=2}^{n}\left(\ell^{k}-1\right)($ see $[3 ;$ p. 155]), $(13),(14 ; m=3)$ and $(6 ; m=1)$ 
give

$$
\begin{aligned}
& I\left(n, x, 0, n^{-3 / 2} \ln n\right) \\
& =\int_{0}^{n^{-3 / 2} \ln n} \exp \left\{-\sum_{\ell=2}^{n}\left(\frac{\ell^{2}-1}{6} t^{2}+\frac{\ell^{4}-1}{180} t^{4}+\frac{\ell^{6}-1}{2835} t^{6}+O\left(n^{8} t^{8}\right)\right)\right\} \cos \left(\frac{x t n^{3 / 2}}{3}\right) d t \\
& =\int_{0}^{n^{-3 / 2} \ln n} \exp \left\{-\frac{\theta_{2}(n) t^{2}}{6}-\frac{\theta_{4}(n) t^{4}}{180}-\frac{\theta_{6}(n) t^{6}}{2835}+O\left(n^{9} t^{8}\right)\right\} \cos \left(\frac{x t n^{3 / 2}}{3}\right) d t \\
& =\int_{0}^{n^{-3 / 2} \ln n} \exp \left\{-\frac{\theta_{2}(n) t^{2}}{6}-\frac{\theta_{4}(n) t^{4}}{180}-\frac{\theta_{6}(n) t^{6}}{2835}\right\} \cos \left(\frac{x t n^{3 / 2}}{3}\right) d t+O\left(\frac{\ln ^{9} n}{n^{9 / 2}}\right) \\
& =\frac{3}{n^{3 / 2}} \int_{0}^{\ln n / 3} \exp \left(-\frac{u^{2}}{2}\right) \exp \left\{R_{2}(n) u^{2}+R_{4}(n) u^{4}+R_{6}(n) u^{6}\right\} \cos (u x) d u \\
& \quad+O\left(\frac{\ln ^{9} n}{n^{9 / 2}}\right),
\end{aligned}
$$

upon setting $u=n^{3 / 2} t / 3$, where $R_{2}(n)=-3 / 4 n+5 / 4 n^{2}, R_{4}(n)=-9 / 100 n-9 / 40 n^{2}-$ $3 / 20 n^{3}+93 / 200 n^{5}$ and $R_{6}(n)=-9 / 245 n^{2}-9 / 70 n^{3}-9 / 70 n^{4}+3 / 70 n^{6}+123 / 490 n^{8}$. It is readily seen that the error term in (15) is at most e $n^{-9 / 2} \ln ^{9} n$ for all $n \geq 2$ and all real numbers $x$. For $0 \leq u \leq \ln n / 3,(6 ; m=3)$ gives

$$
\begin{aligned}
\exp \{ & \left.R_{2}(n) u^{2}+R_{4}(n) u^{4}+R_{6}(n) u^{6}\right\} \\
& =1+S_{2}(n) u^{2}+S_{4}(n) u^{4}+S_{6}(n) u^{6}+S_{8}(n) u^{8}+O\left(\frac{\ln ^{18} n}{n^{3}}\right),
\end{aligned}
$$

where $S_{2}(n)=-3 / 4 n+5 / 4 n^{2}, S_{4}(n)=-9 / 100 n+9 / 160 n^{2}, S_{6}(n)=603 / 19600 n^{2}$ and $S_{8}(n)=81 / 20000 n^{2}$. Hence, (15) and (16) give

$$
\begin{aligned}
& I\left(n, x, 0, n^{-3 / 2} \ln n\right) \\
& =\frac{3}{n^{3 / 2}} \int_{0}^{\ln n / 3} \exp \left(-\frac{u^{2}}{2}\right)\left\{1+S_{2}(n) u^{2}+S_{4}(n) u^{4}+S_{6}(n) u^{6}+S_{8}(n) u^{8}\right. \\
& \left.+O\left(\frac{\ln ^{18} n}{n^{3}}\right)\right\} \cos (u x) d u+O\left(\frac{\ln ^{9} n}{n^{9 / 2}}\right) \\
& =\frac{3}{n^{3 / 2}} \int_{0}^{\ln n / 3} \exp \left(-\frac{u^{2}}{2}\right)\left\{1+S_{2}(n) u^{2}+S_{4}(n) u^{4}+S_{6}(n) u^{6}+S_{8}(n) u^{8}\right\} \cos (u x) d u \\
& +O\left(\frac{\ln ^{19} n}{n^{9 / 2}}\right) \\
& =\frac{3}{n^{3 / 2}} \int_{0}^{\infty} \exp \left(-\frac{u^{2}}{2}\right)\left\{1+S_{2}(n) u^{2}+S_{4}(n) u^{4}+S_{6}(n) u^{6}+S_{8}(n) u^{8}\right\} \cos (u x) d u \\
& +O\left(\int_{\ln n / 3}^{\infty} \exp \left(-\frac{u^{2}}{4}\right) d u\right)+O\left(\frac{\ln ^{19} n}{n^{9 / 2}}\right)
\end{aligned}
$$


THE ELECTRONiC JOURnAl of COMBinatorics 7 (2000), \#R50

$$
\begin{aligned}
=\frac{3}{n^{3 / 2}} \int_{0}^{\infty} & \exp \left(-\frac{u^{2}}{2}\right)\left\{1+S_{2}(n) u^{2}+S_{4}(n) u^{4}+S_{6}(n) u^{6}+S_{8}(n) u^{8}\right\} \cos (u x) d u \\
& +O\left(\frac{\ln ^{19} n}{n^{9 / 2}}\right)
\end{aligned}
$$

where the last equation follows from (7). The error term in the first equation holds uniformly for all real numbers $x$ by the comments after (15) and, since $|\cos (u x)| \leq 1$, the error term in the second equation holds uniformly for all real numbers $x$ by (16) as does the error term in the third equation involving the integral. Then (8), (10-12) and (17) give

$$
\begin{aligned}
I(n, x)=\frac{3}{n^{3 / 2}}\left\{F_{0}(x)\right. & \left.+S_{2}(n) F_{2}(x)+S_{4}(n) F_{4}(x)+S_{6}(n) F_{6}(x)+S_{8}(n) F_{8}(x)\right\} \\
+ & O\left(\frac{\ln ^{19} n}{n^{9 / 2}}\right),
\end{aligned}
$$

where our error term holds uniformly for all real numbers $x$. Hence, after simplifying (18) we obtain

$$
\begin{aligned}
I(n, x)=3( & \left.\frac{\pi}{2}\right)^{1 / 2} n^{-3 / 2} \mathrm{e}^{-x^{2} / 2}\left\{1-\frac{1}{100 n}\left(9 x^{4}-129 x^{2}+102\right)\right. \\
& +\frac{1}{980000 n^{2}}\left(3969 x^{8}-141282 x^{6}+1340865 x^{4}\right. \\
& \left.\left.-4579480 x^{2}+2259370\right)\right\}+O\left(\frac{\ln ^{19} n}{n^{9 / 2}}\right),
\end{aligned}
$$

where our error term holds uniformly for all real numbers $x$. Our result follows since, apart from the error term, the smallest term in (19) has order of magnitude at least $n^{-4}$ for $x^{2}=x^{2}(n) \leq \ln n$.

We note several consequences of Theorem 1.

Corollary 2. For $x^{2}=x^{2}(n) \leq \ln n$, we have the asymptotic expansion

$$
\begin{aligned}
\frac{b(n, k)}{n !}=6(2 \pi)^{-1 / 2} n^{-3 / 2} e^{-x^{2} / 2}\left\{1-\frac{1}{100 n}\left(9 x^{4}-129 x^{2}+102\right)\right. \\
+\frac{1}{980000 n^{2}}\left(3969 x^{8}-141282 x^{6}+1340865 x^{4}\right. \\
\left.\left.-4579480 x^{2}+2259370\right)\right\}+O\left(\frac{\ln ^{19} n}{n^{9 / 2}}\right) \text { as } n \rightarrow \infty
\end{aligned}
$$

when $2 k=\left(\begin{array}{l}n \\ 2\end{array}\right) \pm x n^{3 / 2} / 3$. We also have the asymptotic expansion

$$
\frac{b(n, k)}{n !}=6(2 \pi)^{-1 / 2} n^{-3 / 2}\left(1-\frac{51}{50 n}+\frac{225937}{98000 n^{2}}\right)+o\left(\frac{1}{n^{7 / 2}}\right) \text { as } n \rightarrow \infty,
$$

provided $2 k=\left(\begin{array}{l}n \\ 2\end{array}\right)+o\left(n^{1 / 2} \ln ^{-3 / 2} n\right)$. In particular, $B(n) / n$ ! has the same asymptotic expansion. 
Proof. The asymptotic expansion for $b(n, k) / n$ ! when $2 k=\left(\begin{array}{l}n \\ 2\end{array}\right) \pm x n^{3 / 2} / 3$ where $x^{2}=$ $x^{2}(n) \leq \ln n$ follows immediately from (3) and Theorem 1. For all $n \geq \mathrm{e}^{141}$ and all real numbers $x,(8)$ and (10-12) give

$$
\left|\int_{n^{-3 / 2} \ln n}^{\pi / 2} \prod_{\ell=2}^{n}\left(\frac{\sin \ell t}{\ell \sin t}\right)\left\{1-\cos \left(\frac{x t n^{3 / 2}}{3}\right)\right\} d t\right| \leq 10 \exp \left(-\frac{\ln ^{2} n}{72}\right) .
$$

For an integer $\ell \geq 2$ and all $t \in[0, \pi / 2 \ell], \sin \ell t / \ell \sin t \in[0,1]$ by induction on $\ell$. Then, for all $n \geq 2$ and all $x \in\left[0, \ln ^{-1} n\right]$, (5) gives

$$
\begin{aligned}
0 & \leq \int_{0}^{n^{-3 / 2} \ln n} \prod_{\ell=2}^{n}\left(\frac{\sin \ell t}{\ell \sin t}\right)\left\{1-\cos \left(\frac{x t n^{3 / 2}}{3}\right)\right\} d t \\
& \leq \int_{0}^{n^{-3 / 2} \ln n} \frac{x^{2} t^{2} n^{3}}{18} d t=\frac{x^{2} \ln ^{3} n}{54 n^{3 / 2}} .
\end{aligned}
$$

Hence, for all $n \geq \mathrm{e}^{141}$ and all $x \in\left[0, \ln ^{-1} n\right],(20)$ and (21) give

$$
|I(n, 0)-I(n, x)| \leq \frac{x^{2} \ln ^{3} n}{54 n^{3 / 2}}+10 \exp \left(-\frac{\ln ^{2} n}{72}\right)
$$

Assume $\left(\begin{array}{l}n \\ 2\end{array}\right)$ is even (odd $\left(\begin{array}{l}n \\ 2\end{array}\right)$ is similar) and $n \geq \mathrm{e}^{141}$. Let $\ell:=\left\lfloor\left(\begin{array}{l}n \\ 2\end{array}\right) / 2+n^{3 / 2} / 6 \ln n\right\rfloor$ so that $2 \ell=\left(\begin{array}{l}n \\ 2\end{array}\right)+x n^{3 / 2} / 3$ with $x \in\left[0, \ln ^{-1} n\right]$. For $\left(\begin{array}{l}n \\ 2\end{array}\right) \leq 2 k \leq 2 \ell$, log concavity of the $b(n, k)$ implies

$$
b\left(n, \frac{\left(\begin{array}{c}
n \\
2
\end{array}\right)}{2}\right) \geq b(n, k) \geq b(n, \ell)
$$

so that (3) and (22) give

$$
\frac{2}{\pi} I(n, 0) \geq \frac{b(n, k)}{n !} \geq \frac{2}{\pi} I(n, 0)-\frac{x^{2} \ln ^{3} n}{27 \pi n^{3 / 2}}-\frac{20}{\pi} \exp \left(-\frac{\ln ^{2} n}{72}\right) .
$$

Hence, Theorem 1 gives

$$
\frac{b(n, k)}{n !}=6(2 \pi)^{-1 / 2} n^{-3 / 2}\left(1-\frac{51}{50 n}+\frac{225937}{98000 n^{2}}\right)+o\left(\frac{1}{n^{7 / 2}}\right),
$$

for $2 k=\left(\begin{array}{l}n \\ 2\end{array}\right)+o\left(n^{1 / 2} \ln ^{-3 / 2} n\right)$.

Remark. We can replace the $o\left(n^{-7 / 2}\right)$ error term in the asymptotic expansion of $B(n) / n$ ! with $O\left(n^{-9 / 2} \ln ^{19} n\right)$.

The following extension of Theorem 1 (the case $m=3$ ) giving a complete asymptotic expansion of $I(n, x)$ can be immediately read out of its proof. 
Theorem 3. Fix an integer $m \geq 2$. For $x^{2}=x^{2}(n) \leq \ln n$, we have the asymptotic expansion

$$
\begin{gathered}
I(n, x)=3\left(\frac{\pi}{2}\right)^{1 / 2} n^{-3 / 2} e^{-x^{2} / 2}\left\{1+\sum_{q=1}^{2 m-2}(-2)^{-q} S_{2 q}(n) H_{2 q}\left(2^{-1 / 2} x\right)\right\} \\
+O\left(\frac{\ln ^{2 m^{2}+1} n}{n^{m+3 / 2}}\right) \text { as } n \rightarrow \infty .
\end{gathered}
$$

(The $S_{2 q}(n)$ are defined in the proof.)

Proof. For $2 \leq \ell \leq n$ and $t \in\left[0, n^{-1}\right],(13)$ and (14) give

$$
\ln \left(\frac{\sin \ell t}{\ell \sin t}\right)=\sum_{k=1}^{m} c_{2 k}\left(\ell^{2 k}-1\right) t^{2 k}+O\left(n^{2 m+2} t^{2 m+2}\right),
$$

where $c_{2 k}:=(-4)^{k} B_{2 k} /(2 k)(2 k) !<0$, while,

$$
0 \leq \theta_{2 k}(n)=\sum_{\ell=2}^{n}\left(\ell^{2 k}-1\right)=\frac{1}{2 k+1} \sum_{j=0}^{2 k} B_{j}\left(\begin{array}{c}
2 k+1 \\
j
\end{array}\right)(n+1)^{2 k+1-j}-n .
$$

Hence, $(23)$ and $(6 ; m=1)$ give

$$
\begin{aligned}
& I\left(n, x, 0, n^{-3 / 2} \ln n\right) \\
& =\frac{3}{n^{3 / 2}} \int_{0}^{\ln n / 3} \exp \left\{\sum_{k=1}^{m} 9^{k} c_{2 k} \theta_{2 k}(n) u^{2 k} n^{-3 k}\right\} \cos (u x) d u+O\left(\frac{\ln ^{2 m+3} n}{n^{m+3 / 2}}\right) \\
& =\frac{3}{n^{3 / 2}} \int_{0}^{\ln n / 3} \exp \left(-\frac{u^{2}}{2}\right) \exp \left\{R_{2}(n) u^{2}+\cdots+R_{2 m}(n) u^{2 m}\right\} \cos (u x) d u \\
& \quad+O\left(\frac{\ln ^{2 m+3} n}{n^{m+3 / 2}}\right),
\end{aligned}
$$

where $R_{2}(n)=-3 / 4 n+5 / 4 n^{2}$ and, for $2 \leq k \leq m$,

$$
R_{2 k}(n):=\frac{(-36)^{k} B_{2 k}}{(2 k)(2 k+1) !} \sum_{j=0}^{2 k} B_{j}\left(\begin{array}{c}
2 k+1 \\
j
\end{array}\right) n^{-3 k}(n+1)^{2 k+1-j}-\frac{(-36)^{k} B_{2 k}}{(2 k)(2 k) !} n^{-3 k+1} .
$$

The error term in (24) holds uniformly for all real numbers $x$. For $2 \leq k \leq m \leq n-1$, crude estimates (see $[1 ;$ p. 805]) give

$$
\left|R_{2 k}(n)\right| \leq 60(2 k+1) ! n^{-k+1}
$$


(in fact, $R_{2 k}(n)$ involves $n^{-k+1}$ and smaller integer powers of $n$ ). For all $n \geq m+1$ and all $0 \leq u \leq \ln n / 3$, (25) gives

$$
\left|R_{2}(n) u^{2}+\cdots+R_{2 m}(n) u^{2 m}\right| \leq m(2 m+1) !\left(\frac{\ln ^{2 m} n}{n}\right) .
$$

Hence, (6) and (26) give

$$
\exp \left\{R_{2}(n) u^{2}+\cdots+R_{2 m}(n) u^{2 m}\right\}=1+\sum_{q=1}^{2 m-2} S_{2 q}(n) u^{2 q}+O\left(\frac{\ln ^{2 m^{2}} n}{n^{m}}\right),
$$

where $S_{2 q}(n)$ is that part of

$$
\sum_{r=1}^{m-1} \sum_{\begin{array}{c}
\left(e_{2}, \ldots, e_{2 m}\right) \in \mathbb{N}^{m} \\
e_{2}+\cdots+e_{2 m}=r \\
2 e_{2}+\cdots+2 m e_{2 m}=2 q
\end{array}} \frac{R_{2}^{e_{2}}(n) \cdots R_{2 m}^{e_{2 m}}(n)}{e_{2} ! \cdots e_{2 m} !}
$$

involving only $n^{-1}, \ldots, n^{-m+1}$ upon expansion. Here $R_{2}^{e_{2}}(n) \cdots R_{2 m}^{e_{2 m}}(n)$ involves $n^{-\left(e_{2}+\cdots+(m-1) e_{2 m}\right)}=n^{-\left(q-r+e_{2}\right)}$ and smaller integer powers of $n$ while $q-r+e_{2} \geq m$ if $q \geq 2 m-1$. Then, (24) and (27) give

$$
\begin{aligned}
& I\left(n, x, 0, n^{-3 / 2} \ln n\right) \\
& =\frac{3}{n^{3 / 2}} \int_{0}^{\ln n / 3} \exp \left(-\frac{u^{2}}{2}\right)\left\{1+\sum_{q=1}^{2 m-2} S_{2 q}(n) u^{2 q}\right\} \cos (u x) d u+O\left(\frac{\ln ^{2 m^{2}+1} n}{n^{m+3 / 2}}\right) \\
& =\frac{3}{n^{3 / 2}} \int_{0}^{\infty} \exp \left(-\frac{u^{2}}{2}\right)\left\{1+\sum_{q=1}^{2 m-2} S_{2 q}(n) u^{2 q}\right\} \cos (u x) d u+O\left(\frac{\ln ^{2 m^{2}+1} n}{n^{m+3 / 2}}\right),
\end{aligned}
$$

where our error term holds uniformly for all real numbers $x$. Hence, after simplifying, (8), (10-12) and (28) give

$$
\begin{gathered}
I(n, x)=3\left(\frac{\pi}{2}\right)^{1 / 2} n^{-3 / 2} \mathrm{e}^{-x^{2} / 2}\left\{1+\sum_{q=1}^{2 m-2}(-2)^{-q} S_{2 q}(n) H_{2 q}\left(2^{-1 / 2} x\right)\right\} \\
+O\left(\frac{\ln ^{2 m^{2}+1} n}{n^{m+3 / 2}}\right),
\end{gathered}
$$

where our error term holds uniformly for all real numbers $x$. Our result follows since, apart from the error term, the smallest term in (29) has order of magnitude at least $n^{-m-1}$ for $x^{2}=x^{2}(n) \leq \ln n$.

As a consequence of Theorem 3, we have a complete asymptotic expansion for $b(n, k) / n$ ! when $2 k=\left(\begin{array}{l}n \\ 2\end{array}\right) \pm x n^{3 / 2} / 3$ where $x^{2}=x^{2}(n) \leq \ln n$, as well as for $B(n) / n$ ! when $\left(\begin{array}{l}n \\ 2\end{array}\right)$ is even. 
Corollary 4. Fix an integer $m \geq 2$. For $x^{2}=x^{2}(n) \leq \ln n$, we have the asymptotic expansion

$$
\begin{gathered}
\frac{b(n, k)}{n !}=6(2 \pi)^{-1 / 2} n^{-3 / 2} e^{-x^{2} / 2}\left\{1+\sum_{q=1}^{2 m-2}(-2)^{-q} S_{2 q}(n) H_{2 q}\left(2^{-1 / 2} x\right)\right\} \\
+O\left(\frac{\ln ^{2 m^{2}+1} n}{n^{m+3 / 2}}\right) \text { as } n \rightarrow \infty,
\end{gathered}
$$

when $2 k=\left(\begin{array}{c}n \\ 2\end{array}\right) \pm x n^{3 / 2} / 3$. In particular, we have the asymptotic expansion

$$
\frac{B(n)}{n !}=6(2 \pi)^{-1 / 2} n^{-3 / 2}\left\{1+\sum_{q=1}^{2 m-2} 2^{-q} \frac{(2 q) !}{q !} S_{2 q}(n)\right\}+O\left(\frac{\ln ^{2 m^{2}+1} n}{n^{m+3 / 2}}\right) \text { as } n \rightarrow \infty
$$

when $\left(\begin{array}{l}n \\ 2\end{array}\right)$ is even.

In the following table we compare the exact value of $B(n) / n$ ! (found by expanding the generating function for the $b(n, k)$ ) with the approximations (given by Corollary 4 for $m=2,3)$ for $n=40$ and 80 .

$$
B(40) / 40 \text { ! } B(80) / 80 \text { ! }
$$

$\begin{array}{ccc}\text { Exact Value } & 0.009233258744992 \cdots & 0.003303747524408 \cdots \\ \text { Approximation }(m=2) & 0.009220472410157 \cdots & 0.003302581000634 \cdots \\ \text { Relative Error } & 0.138481 \% & 0.035309 \% \\ \text { Error as a function of } n & 40-3.05435 \cdots & 80-3.11761 \cdots \\ \text { Approximation }(m=3) & 0.009234106075478 \cdots & 0.003303786057784 \cdots \\ \text { Relative Error } & 0.009176 \% & 0.001166 \% \\ \text { Error as a function of } n & 40-3.79008 \cdots & 80^{-3.89585 \cdots}\end{array}$

Acknowledgement. I wish to thank the referee for numerous comments and suggestions which have led to a substantially improved paper. 
THE ELECTRONiC Journal of COMBINATORICs 7 (2000), \#R50

\section{References}

[1] M. Abramowitz and I.A. Stegun, Eds., Handbook of Mathematical Functions with Formulas, Graphs and Mathematical Tables, Dover Publications, New York, 1966.

[2] E.A. Bender, Central and Local Limit Theorems Applied to Asymptotic Enumeration, J. Combinatorial Theory A 15 (1973), 91-111.

[3] L. Comtet, Advanced Combinatorics, D. Reidel, Boston, 1974.

[4] N.N. Lebedev, Special Functions and Their Applications, Dover Publications, New York, 1972.

[5] T. Muir, On a Simple Term of a Determinant, Proc. Royal Society Edinburg 21 (1898-9), 441-477. 